\title{
Microscopic picture of molecular double doping
}

\author{
Thomas Bathe, ${ }^{1}$ Chuan-Ding Dong, ${ }^{1}$ and Stefan Schumacher ${ }^{1,2, *}$ \\ ${ }^{1}$ Department of Physics and Center for Optoelectronics and Photonics Paderborn (CeOPP), \\ Paderborn University, Warburger Strasse 100, 33098 Paderborn, Germany \\ ${ }^{2}$ College of Optical Sciences, University of Arizona, Tucson, Arizona 85721, USA
}

(Dated: October 15, 2021)

\begin{abstract}
Double doping, in which a single dopant molecule induces two charge carriers in an organic semiconductor (OSC), was recently experimentally observed and promises to enhance the efficiency of molecular doping. Here we present a theoretical investigation of p-type molecular double doping in a CN6-CP:bithiophene-thienothiophene OSC system. Our analysis is based on density functional theory (DFT) calculations for the electronic ground state. In a molecular complex with two OSC oligomers and one CN6-CP dopant molecule we explicitly demonstrate double integer charge transfer and find formation of two individual polarons on the OSC molecules and a di-anion dopant molecule. We show that the vibrational modes and related infrared absorption spectrum of this complex can be traced back to those of the charged dopant and OSC molecules in their isolated forms. The near-infrared optical absorption spectrum calculated by time-dependent DFT shows both features of typical intra-molecular polaron excitations and weak inter-molecular charge transfer excitations associated with the doping-induced polaron states.
\end{abstract}

\section{INTRODUCTION}

Molecular doping is known to be able to enhance the conductivity of organic semiconductors (OSC) by orders of magnitude, and is of crucial importance for their applications. ${ }^{1-3}$ While different doping methods are being actively developed, ${ }^{4-6}$ the efficiency of molecular doping is generally still much lower than the doping achieved in inorganic semiconductors and the mechanisms of molecular doping on a microscopic level are often not fully understood. ${ }^{7-10}$ Improving the doping efficiency is an important aspect of optimizing doped OSC systems. From a microscopic point of view the doping efficiency can for example be limited by aggregation of dopant molecules, which hampers the effective contact between dopant and host, or by the formation of trapped charge transfer states which can not contribute to the conductivity. ${ }^{11-13}$ In addition, improving the conductivity by increasing the dopant concentration often leads to undesirable structural disorder in the OSC film. ${ }^{14,15}$ In this context, it would be beneficial if each single dopant molecule could induce more than one charge into the host OSC material. Recently, this avenue was explored successfully and p-type double doping was realized. ${ }^{16}$ There, each dopant molecule accepted up to two electrons, inducing two effective charges into the copolymer $\mathrm{p}\left(\mathrm{g}_{4} 2 \mathrm{~T}\right.$ TT), substantially enhancing electric transport. This observation, while invoking the general interest in doubledoping, also calls for a more detailed understanding of the underlying mechanisms, as well as of material and parameter dependencies. For example further details of the charge formation such as formation of individual polarons and/or bi-polarons upon double-doping are of significant relevance for the transport properties of the resulting doped system. ${ }^{17-22}$

In this work, we investigate a simultaneous charge transfer between two $\mathrm{p}\left(\mathrm{g}_{4} 2 \mathrm{~T}-\mathrm{TT}\right)$ conjugated polymer (oligomer) chains in a close arrangement and a single
CN6-CP p-type dopant molecule as illustrated in Figure 1(a). Our study is firmly based on density functional theory (DFT) calculations taking into account inter-molecular van-der Waals interactions and dielectric environment. While our approach includes the possibility for other electronic processes to occur, ${ }^{21}$ the results clearly show double p-type doping in the form of a double integer charge transfer from the conjugated chains onto the dopant molecule: a CN6-CP di-anion is formed along with two individual singly charged polarons, one on each polymer chain. The vibrational IR spectrum we calculate for the CN6-CP:2p $\left(\mathrm{g}_{4} 2 \mathrm{~T}-\mathrm{TT}\right)$ complex with double doping can clearly be traced back to those of individual polarons and a $\mathrm{CN6}-\mathrm{CP}$ di-anion. We further demonstrate that the doping induced charges oscillate in space upon geometry displacement along the vibrational normal modes with highest intensities in the IR spectrum. ${ }^{25}$ The near-infrared optical absorption spectrum of the complex contains the typical features of intramolecular polaron excitations which in our calculations are distinct from the low-intensity inter-molecular charge transfer excitations also observed.

\section{SYSTEM AND METHODS}

The OSC studied in this work is a bithiophenethienothiophene copolymer $\mathrm{p}\left(\mathrm{g}_{4} 2 \mathrm{~T}-\mathrm{TT}\right)$ for which double doping was recently demonstrated experimentally. ${ }^{16}$ To reduce the computational cost, the tetraethylene glycol side chains are replaced by methoxy groups (Figure 1a). In the present study the OSC is described by a finite length oligomer consisting of three repeat units. In this first theoretical study on molecular double doping we use the strong CN6-CP p-type dopant. Geometry optimization and calculation of the electronic ground state are based on density funtional theory (DFT) as implemented in the Gaussian 09 program suite ${ }^{26}$. To allow 
a)

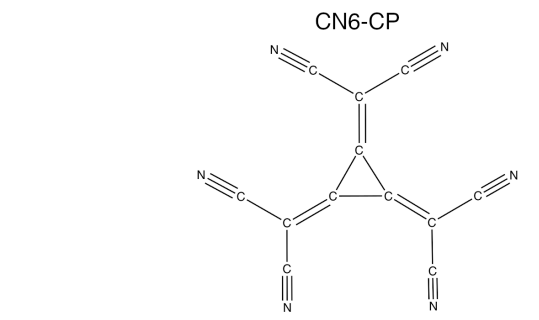

OSC

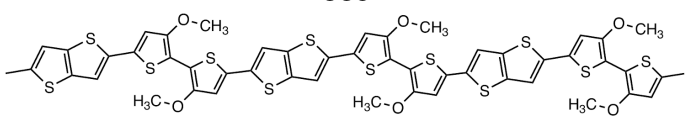

b)

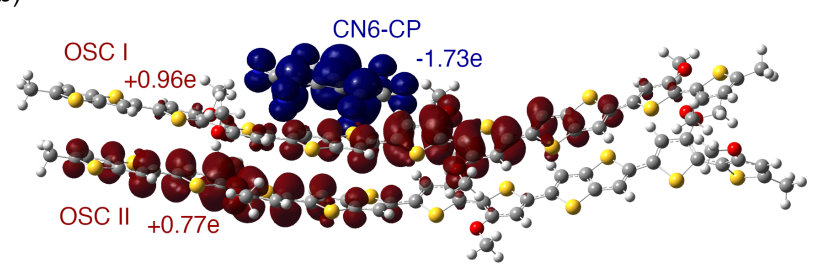

C)

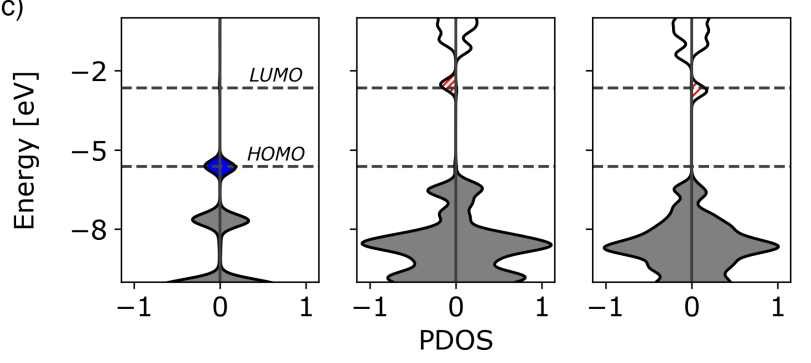

FIG. 1. (a) Chemical structures of dopant molecule CN6-CP and OSC material $\mathrm{p}\left(\mathrm{g}_{4} 2 \mathrm{~T}-\mathrm{TT}\right)$. (b) Molecular complex with optimized geometry. Doping induced charges are represented by molecular orbitals of the highest occupied states (blue) and the lowest unoccupied states (red). Resulting net charge on the three molecular entities is also given. (c) Electronic density of states of the molecules in the complex of panel (b), for the dopant molecule (left), OSC oligomer 1 (center), and OSC oligomer 2 (right). Doping induced DOS corresponding to the orbitals shown in (b) is marked with color. Occupied states are marked with shading.

for flexibility in the electronic processes that may occur in the OSC-dopant complexes, we use broken symmetry (BS) DFT calculations, where the $\alpha$ and $\beta$ orbitals are treated separately, following our and other researchers' previous studies. ${ }^{20-24}$ It should be noted there the $\alpha$ and $\beta$ channel can actually have mixed spin components in the BS-DFT calculations. The range separated $\omega \mathrm{B} 97 \mathrm{X}-$ $\mathrm{D}$ functional that also includes the dispersion correction (van-der Waals interaction) is used throughout this work along with the $6-31 \mathrm{G}^{*}$ basis set. In our previous work, it was shown that the $\omega \mathrm{B} 97 \mathrm{X}-\mathrm{D}$ functional gives a similar description of charge transfer processes as the CAMB3LYP functional. ${ }^{21}$ To account for the effects of neighbouring molecules in an actual OSC environment, a dielectric background of $\varepsilon=3$ is used within the polarizable continuum model. We note that we did not observe double doping with $\varepsilon=2$ or smaller values in the calculations. The charge analysis and the projected density of states (PDOS) calculations are performed using the software Multiwfn. ${ }^{27}$

\section{RESULTS}

\section{A. Double-doping}

The molecular constituents and the molecular complex studied in the present work are depicted in Figure 1 (a) and (b). Panel (c) of Figure 1 shows the electronic density of states projected onto each molecular entity. The calculated HOMO level of an isolated CN6-CP molecule and that of a $\mathrm{p}\left(\mathrm{g}_{4} 2 \mathrm{~T}-\mathrm{TT}\right)$ oligomer are at $-10.44 \mathrm{eV}$ and $-6.22 \mathrm{eV}$, respectively, with the latter being significantly higher than the former, such that p-type doping is efficiently driven in this system, and even double doping is possible as discussed in the following. In this context, it is important to note that absolute values of orbital energies strongly depend on the DFT functional used, only relative ordering and changes are relevant for the discussions in the present work. For the CN6-CP:2p $\left(\mathrm{g}_{4} 2 \mathrm{~T}-\mathrm{TT}\right)$ complex, as shown by the molecular orbitals in Figure 1(b) and the projected density of states in Figure 1(c), both $\alpha$ and $\beta$ HOMO are located on the dopant while one LUMO is located on oligomer $I$ and the other is located on oligomer $I I$. The spatial relocation of HOMO electrons to the CN6-CP site in the complex already indicates double doping. To quantify the amount of charge transferred, we also calculate the Hirshfeld charges of each molecular entity in the complex. With a charge of $-1.73 \mathrm{e}$ on the $\mathrm{CN} 6-\mathrm{CP}$ it is evident that di-anion formation has occured as the result of an integer charge transfer of two electrons. The OSC $I$ and OSC $I I$ oligomers are charged with $+0.96 \mathrm{e}$ and $+0.77 \mathrm{e}$, respectively. This, together with the HOMOs residing on the dopant, clearly evidences double doping where each oligomer donates one electron to the $\mathrm{CN} 6-\mathrm{CP}$ molecule, giving rise to di-anion dopant formation. The molecular states corresponding to the two doping induced polarons in the doubly doped complex are close in energy both located at about -2.65 eV, cf. Figure 1(c). These two polaron states show a certain spatial shift to opposite directions along the conjugated chains, cf. Figure 1(b). This behavior is different from that of an isolated polaron and manifests the repulsive interaction between the two polarons with equal charge. We note that in these calculations we did not find the formation of a bi-polaron or side-by-side polaron state on a single polymer chain. ${ }^{22,28}$

\section{B. Vibrational modes}

Next, we turn our attention to the vibrational modes of the complex with double doping. In particular, the IR absorption spectrum of the complex is calculated and com- 


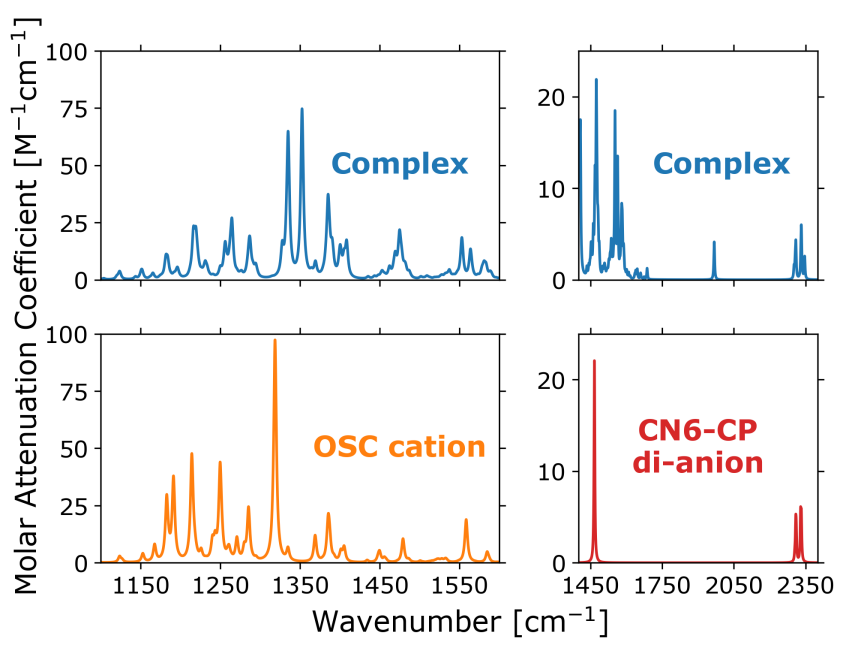

FIG. 2. Vibrational spectra showing the fingerprints of doping induced charges. Top panels show the IR spectrum of the complex shown in Figure 1 (note the different scaling of yaxes in left and right panels). Bottom left shows spectrum of a singly charged OSC cation. Bottom right shows spectrum of a doubly charged CN6-CP di-anion.

pared with the spectra of the individual oligomer cation and the CN6-CP di-anion as shown in Figure 2. While not being identical because of the system complexity, the vibrational spectral features of the CN6-CP di-anion can also clearly be identified in the spectrum of the complex. The same is true for the oligomer cation. However, in that case besides a generally strong resemblance a one to one correspondance with the IR spectrum of the complex is not found due to the large number of vibrational modes contributing to the spectrum. Nevertheless, in particular the spectrally dominant mode in the oligomer cation at $1319 \mathrm{~cm}^{-1}$ can also be identified in the complex. The corresponding modes are slightly red-shifted (at 1334 and $1352 \mathrm{~cm}^{-1}$ ) in the complex and appear twice due to the two oligomers contributing. These two peaks in the Complex IR spectrum can be identified as the typical intense vibrational modes (IVMs) observed in the presence of polarons. $^{25,29}$

To illustrate this aspect further, we also study the polaron movement when the molecular geometry is displaced along thoses vibrational normal modes associated with the IVMs. For each displacement, we then perform single point calculations of the new geometries using the wavefunction of the optimized geometry as an initial guess. Results are summarized in Figure 3. Note that due to the charge transfer present in the complex, the static electric dipole moment is non-zero in the relaxed state. The change of the dipole moments caused by the displacement along both IVMs is shown in Figure $3(\mathrm{a}, \mathrm{b})$. For both IVMs, we see that the dipole moment changes mainly in one direction, which corresponds to the movement of the polaron along the backbone of each oligomer, while the change in the other directions is a)

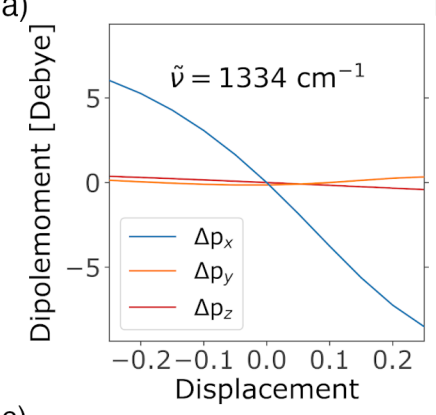

c)
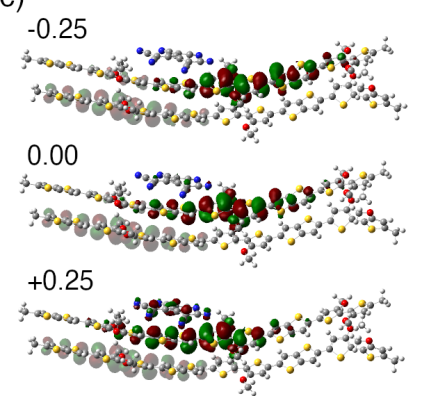

b)

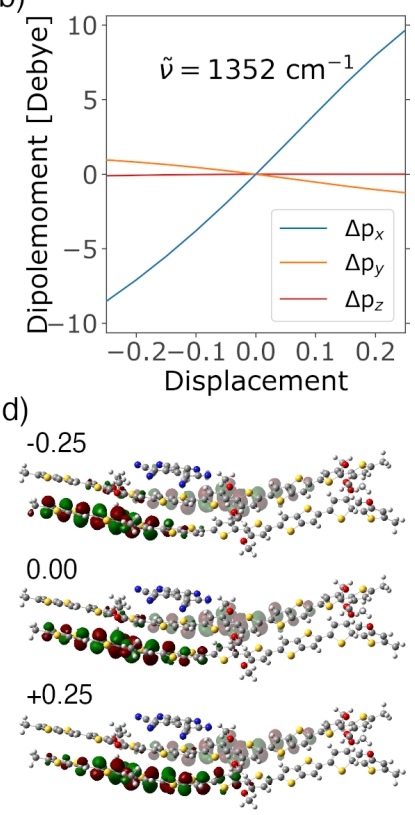

FIG. 3. Change in static dipole moment by displacement along the normal coordinates of the most intense vibrational modes (IVMs) in Figure 2. (a) and (b) show the vector components of the static dipole moment versus displacement. (c) and (d) show the molecular orbitals associated with the doping induced charges on the two OSC molecules for three different displacements as indicated along the IVM normal coordinates, illustrating the spatial oscillation of the induced charges with displacement.

only minor. The molecular orbitals of the polarons for the two largest displacements of both IVMs and the relaxed geometries are also shown in Figure 3(c,d). For each IVM we see that only one polaron is displaced upon displacement such that it oscillates around its relaxed position, whereas the other polaron is not much affected. Therefore, each of the two IVMs corresponds to the oscillation of one of the polarons, respectively. This confirms that the two large peaks in the vibrational spectrum of the complex are related to the two positive charges induced on the conjugated oligomers in the complex.

\section{Optical spectra}

Next, we study the optical excitations of the complex. For this purpose it is instructive to first take a look at the molecular energy levels in the electronic ground state of the individual molecular entities and the complex as informed by our calculations and sketched in Figure 4. In this simplified single particle picture, in the complex, transfer of one electron from each of the oligomers onto the dopant molecule occurs, one in the $\alpha$ and one in the $\beta$ spin channel. This results in a doubly occupied orbital on the dopant, forming the HOMO of the complex, and leaving an unoccupied orbital, i.e., polaron, on each of the 
a)

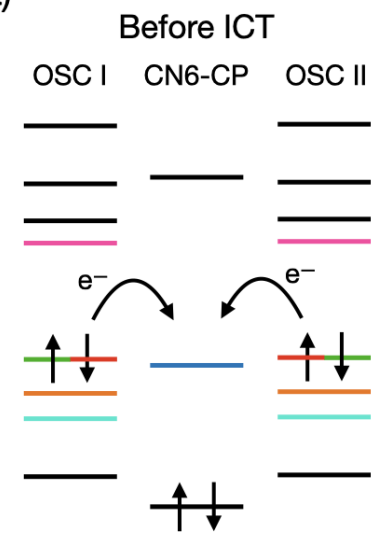

b)

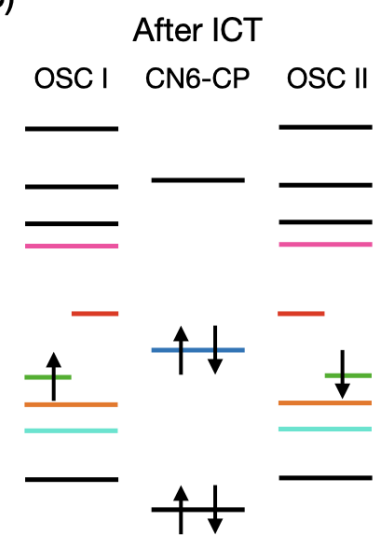

c)

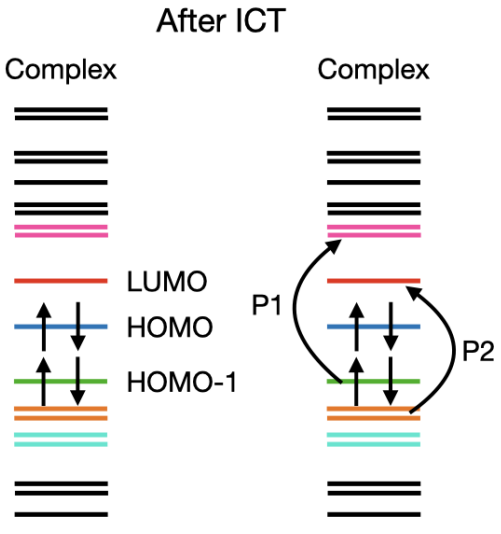

FIG. 4. Schematic picture of energy levels in the complex of Figure 1. (a) Levels of isolated molecular constituents. Double charge transfer from OSC molecules to dopant is indicated. (b) Energy levels of the molecules in the complex after double charge transfer. (c) Resulting energy levels of the complex with highest occupied molecular orbital indicated. Doping induced states, LUMOs, lie inside the OSC gap. Also illustrated are the optically induced electronic excitations in the complex, with P1 and P2 polaron excitations indicated.

oligomers. These two unpaired unoccupied orbitals must have different spin. Consequently the two remaining unpaired electrons on the oligomers also have different spin. In the complex these two electrons occupy the HOMO-1 orbital. The two doping induced unoccupied orbitals on the oligomers, i.e., the polarons, form the LUMO of the complex. As a consequence, the $\alpha$ and $\beta$ components of both LUMO and HOMO-1 levels are nearly degenerate in energy but are spatially well separated and reside on the different oligomers of the complex, as illustrated for the LUMO in Figure 1(c). As also shown in the figure, both spin channels of the HOMO level are localized on the CN6-CP molecule.

Based on the level scheme developed in Figure 4, also the possible electronic excitations can be qualitatively understood and categorized as illustrated in Figure 4(c). Compared to the charge neutral OSC, the formation of in-gap polaron states leads to new features also in the optical excitation spectrum. Among those excitations from occupied into unoccupied electronic orbitals that do not involve any significant charge transfer between molecular constituents of the complex, we call those excitations from HOMO-1 to LUMO+1 and higher interband excitations and label the corresponding spectral features as P1. Those starting in lower-lying occupied levels and ending in the in-gap polaron state, or LUMO, we call intraband excitations and label them with $\mathrm{P} 2$. In addition to the dominant polaron excitations discussed above and sketched in Figure 4(c), additional electronic excitations are possible between molecular entities in the complex that however show significant charge transfer character and thus carry relatively low oscillator strength.

The picture of electronic states and electronic excitations developed in Figure 4 is well supported by the optical absorption spectra shown in Figure 5 and calculated using TD-DFT. The calculated optical absorption spectrum of the complex is plotted in Figure 5(a), including
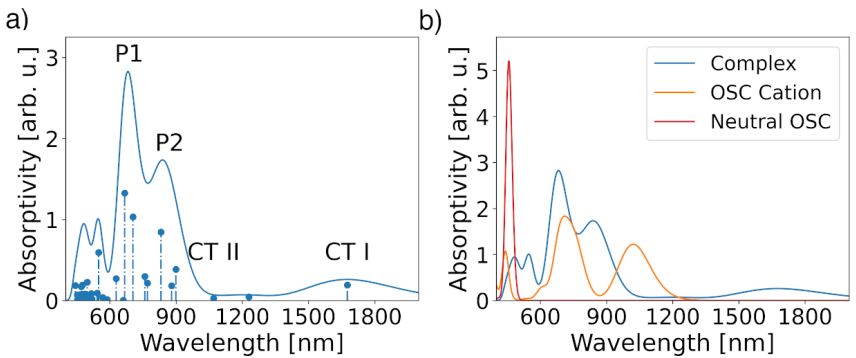

FIG. 5. Optical absorption spectra. (a) Spectrum of the complex of Figure 1 with double doping. P1 and P2 polaron excitations are marked as well as selected charge transfer excitation that occur in the complex. A corresponding stick spectrum for the underlying electronic transitions is also included. (b) Spectra of neutral and singly positively charged OSC oligomer. Also included is the complex spectrum of panel (a) for comparison.

the first 30 excitation as indicated in the underlying stick spectrum. The spectrum features two prominent peaks at around $670 \mathrm{~nm}$ and $830 \mathrm{~nm}$ which can be traced back to the polaron $\mathrm{P} 1$ and $\mathrm{P} 2$ peaks, respectively. Figure 5(b) shows the comparison of the optical absorption spectra of neutral and singly positively charged OSC oligomer and the complex.

For interpretation of the optical absorption spectrum of Figure 5(a), Figure 6(a) gives the natural transition orbitals of select important electronic excitations. Transitions in Figure 6(a) and (b) are of the P2 polaron type. Figure 6(b) shows the first NTO pair of both $\alpha$ and $\beta$ spin for the excitation at $832 \mathrm{~nm}$. Both contributions can be identified as P2 transitions and the involved LUNTO profiles show typical features of the polaron orbitals. The $\beta$ contribution is intramolecular and the $\alpha$ contribution is intermolecular. More specifically, the $\beta$ contribution 
a)

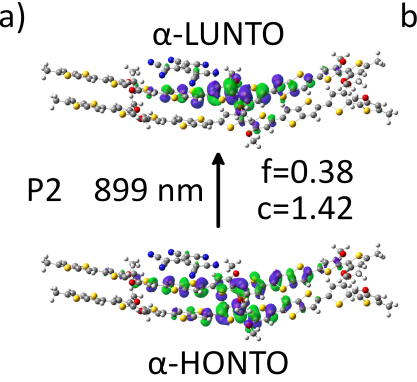

c)
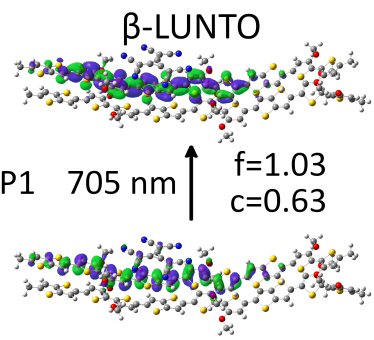

$\beta$-HONTO

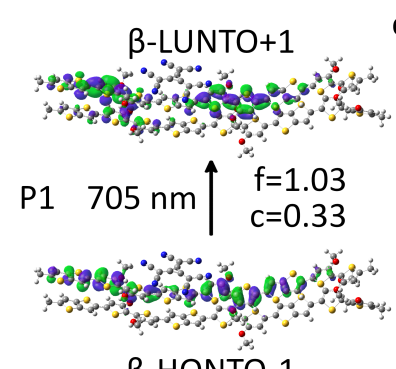

$\beta$-HONTO-1

e)

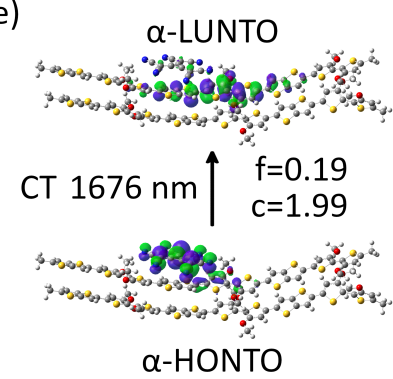

b)
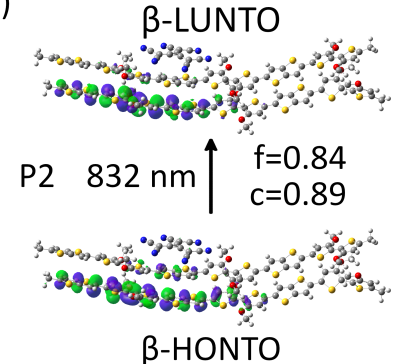

$\beta$-HONTO
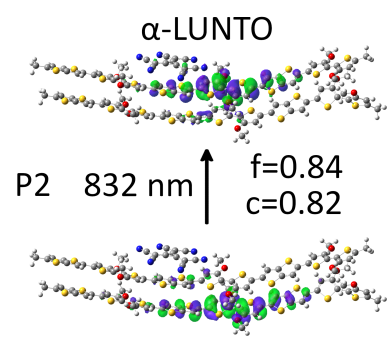

$\alpha$-HONTO

d)

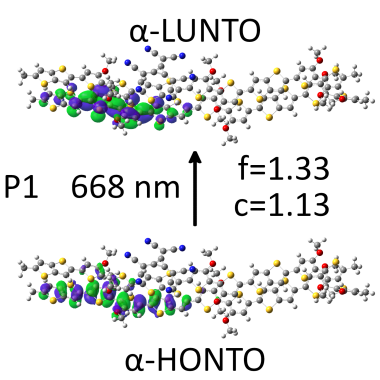

f)

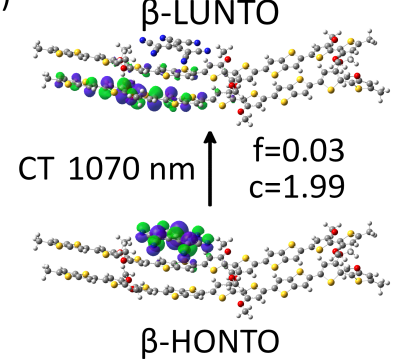

FIG. 6. Natural transition orbitals (NTOs) to support interpretation of optical spectra in Figure 5. (a)-(f) show dominant NTO pairs of select doping induced transitions. Transitions in (a) and (b) show the characteristics of P2 polaron excitations, (c) and (d) those of P1 polaron excitations. (e) and (f) possess dominant charge transfer (CT) character with excitation from dopant into doping induced levels on the OSC molecules. Wavelength, oscillator strength, f, and NTO coefficients, c, are given for each transition.

resembles the $\mathrm{P} 2$ transition that can be found for a single oligomer cation and the $\alpha$ contribution has clear CT character which is enabled by the proximity of the second conjugated chain. In the same band of the spectrum in Figure 5(a), the excitation at $899 \mathrm{~nm}$ is also a P2 transition, dominated by only one NTO pair in the $\alpha$ spin channel as shown in Figure 6(a). This transition also has a slightly mixed intra- and intermolecular character.

The strongest peak in the spectrum in Figure 5(a) stems from the P1 polaron excitations on either doped oligomer. These excitations are each well described by NTO pairs located on a single conjugated chain as shown in Figs. 6(c) and (d). Both excitations show clear $\pi$ $\pi^{*}$ character and constitute the P1 polaron transitions. In addition, the excitations at around $770 \mathrm{~nm}$ can also be identified as P1 transitions whereas the excitation at $880 \mathrm{~nm}$ features both a P2 and an intermolecular contribution (not shown).

A comparison with the neutral OSC oligomer and its cation shows that the cation also features two peaks with similar oscillator strengths in a similar wavelength range as shown in Figure 5(b). However, the spectral peaks of the cation are red-shifted compared to the complex. The spectral separation between the P1 and P2 peak is also larger in the cation than in the complex. In the calculated range, the neutral OSC oligomer features only one large peak which is not present in the complex or the cation. A smaller peak at $1676 \mathrm{~nm}$ is only present in the spectrum of the complex and corresponds to a charge transfer excitation from the dopant to the OSC oligomer $I$ as shown in Figure 6(e). The corresponding excitation for the OSC oligomer II is found at $1070 \mathrm{~nm}$ but with the larger spatial separation of NTOs has a very small oscillator as shown in Figure 6(f).

\section{CONCLUSIONS}

In the present work, we show that p-type double doping as a two-fold integer charge transfer process from two conjugated polymer chains onto a single dopant molecule, can be described by ground state broken symmetry DFT calculations. The double doping in the CN6-CP:bithiophene-thienothiophene complex studied is manifested by the formation of a CN6-CP di-anion and two individual positively charged polarons on the conjugated chains, respectively. Also in the presence of the CN6-CP di-anion, the main features of the vibrational IR spectrum of the molecular complex can be identified as the vibrational modes coupled to the two individual polarons. With the doping-induced formation of in-gap polaron states, the optical absorption spectrum of the complex shows signatures of both polaron intraband excitations (P2 polaron peaks) and polaron interband excitation (P1 polaron peaks) occurring on each of the pdoped conjugated chains. Furthermore, inter-molecular charge transfer excitations with low oscillator strength are also found for the closely packed molecular complex.

\section{ACKNOWLEDGEMENT}

We acknowledge funding from the Deutsche Forschungsgemeinschaft through project SCHU 1980/13 and through the Heisenberg program (No. 270619725). 
* stefan.schumacher@uni-paderborn.de

1 Lüssem, B.; Riede, M.; Leo, K. Doping of Organic Semiconductors Phys. Status Solidi A 2013, 210, 9-43.

2 Reineke, S.; Lindner, F.; Schwartz, G.; Seidler, N.; Walzer, K.; Lüssem, B.; Leo, K. White Organic Light-emitting Diodes with Fluorescent Tube Efficiency Nature 2009, 459, 234-238.

3 Lüssem, B.; Tietze, M. L.; Kleemann, H.; Hoßbach, C.; Bartha, J. W.; Zakhidov, A.; Leo, K. Doped Organic Transistors Operating in the Inversion and Depletion Regime Nat. Commun. 2013, 4, 2775 1-6.

4 Yamashita, Y.; Tsurumi, J.; Ohno, M.; Fujimoto, R.; Kumagai, S.; Kurosawa, T.; Okamoto, T.; Takeya, J.; Watanabe, S. Efficient Molecular Doping of Polymeric Semiconductors Driven by Anion Exchange. Nature 2019, 572, 634-638.

5 Yurash, B.; Cao, D. X.; Brus, V. V. et al. Towards Understanding the Doping Mechanism of Organic Semiconductors by Lewis Acids. Nat. Mater. 2019, 18, 1327-1334.

6 Neusser, D.; Malacrida, C.; Kern, M.; Gross, Y. M.; van Slageren, J.; Ludwigs, S. High Conductivities of Disordered P3HT Films by an Electrochemical Doping Strategy. Chem. Mater. 2020, 32, 6003-6013.

7 Jacobs, I. E.; Moulé, A. J. Controlling Molecular Doping in Organic Semiconductors. Adv. Mater. 2017, 29, 1703063 1-39.

8 Salzmann, I.; Heimel, G.; Oehzelt, M.; Winkler, S.; Koch, N. Molecular Electrical Doping of Organic Semiconductors: Fundamental Mechanisms and Emerging Dopant Design Rules. Acc. Chem. Res. 2016, 49, 370-378.

9 Tietze, M. L.; Benduhn, J.; Pahner, P.; Nell, B.; Schwarze, M.; Kleemann, H.; Krammer, M.; Zojer, K.; Vandewal, K.; Leo, K. Elementary Steps in Electrical Doping of Organic Semiconductors. Nat. Commun. 2018, 9, 1182.

10 Schwarze, M.; Gaul, C.; Scholz, R.; Bussolotti, F.; Hofacker, A.; Schellhammer, K. S.; Nell, B.; Naab, B. D.; Bao, Z.; Spoltore, D.; Vandewal, K.; Widmer, J.; Kera, S.; Ueno, N.; Ortmann, F.; Leo, K. Molecular Parameters Responsible for Thermally Activated Transport in Doped Organic Semiconductors. Nature Mater. 2019, 18, 242-248.

11 Tietze, M. L.; Benduhn, J.; Pahner, P.; Nell, B.; Schwarze, M.; Kleemann, H.; Krammer, M.; Zojer, K.; Vandewal, K.; Leo, K. Elementary Steps in Electrical Doping of Organic Semiconductors. Nat. Commun. 2018, 9, 1182.

12 Jacobs, I. E.; Cendra, C.; Harrelson, T. F.; Bedolla Valdez, Z. I.; Faller, R.; Salleo, A.; Moulé A. J. Polymorphism Controls the Degree of Charge Transfer in a Molecularly Doped Semiconducting Polymer. Mater. Horiz. 2018, 5, 655-660.

13 Pingel, P.; Neher, D. Comprehensive Picture of p-type Doping of P3HT with the Molecular Acceptor F4TCNQ. Phys. Rev. B 2013, 87, 115209.

14 Duong, D. T.; Wang, C. C.; Antono, E.; Toney, M. F.; Salleo, A. The Chemical and Structural Origin of Efficient p-type Doping in P3HT. Org. Electron. 2013, 14, 1330-1336.

15 Schlitz, R. A.; Brunetti, F. G.; Glaudell, A. M.; Miller, P. L.; Brady, M. A.; Takacs, C. J.; Hawker, C. J.; Chabinyc,
M. L. Solubility-Limited Extrinsic n-type Doping of a High Electron Mobility Polymer for Thermoelectric Applications. Adv. Mater. 2014, 26, 2825-2830.

16 Kiefer, D.; Kroon, R.; Hofmann, A. I.; Sun, H.; Liu, X.; Giovannitti, A.; Stegerer, D.; Cano, A.; Hynynen, J.; Yu, L.; Zhang, Y.; Nai, D.; Harrelson, T. F.; Sommer, M.; Moulé, A. J.; Kemerink, M.; Marder, S. R.; McCulloch, I.; Fahlman, M.; Fabiano, S.; Müller, C. Double Doping of Conjugated Polymers with Monomer Molecular Dopants Nat. Mater. 2019, 18, 149-155.

17 Wiebeler, C.; Tautz, R.; Feldmann, J.; Von Hauff, E.; Da Como, E.; Schumacher, S. Spectral Signatures of Polarons in Conjugated Copolymers. J. Phys. Chem. B 2013, 117, 4454-4460.

18 Voss, M. G.; Reddy Challa, J.; Tyler Scholes, D.; Yee, P. Y.; Wu, E. C.; Liu, X.; Park, S. J.; León Ruiz, O.; Subramaniyan, S.; Chen, M.; et al. Driving Force and Optical Signatures of Bipolaron Formation in Chemically Doped Conjugated Polymers Adv. Mater. 2020, 33, 2000228.

19 Tautz, R.; Da Como, E.; Wiebeler, C.; Soavi, G.; Dumsch, I.; Fröhlich, N.; Grancini, G.; Allard, S.; Scherf, U.; Cerullo,G.; Schumacher, S.; Feldmann, J. Charge Photogeneration in Donor-Acceptor Conjugated Materials: Influence of Excess Excitation Energy and Chain Length. J. Am. Chem. Soc. 2013, 135, 4282-4290.

20 Di Nuzzo, D.; Fontanesi, C.; Jones, R.; Allard, S.; Dumsch, I.;Scherf, U.; Von Hauff, E.; Schumacher, S.; Da Como, E. How Intermolecular Geometrical Disorder Affects the Molecular Doping of Donor-Acceptor Copolymers. Nat. Commun. 2015, 6, 6460.

21 Dong, C.-D.; Schumacher, S. Molecular Doping of PCPDT-BT Copolymers: Comparison of Molecular Complexes with and without Integer Charge Transfer. J. Phys. Chem. C 2019, 123, 30863-30870.

22 Dong, C.-D.; Schumacher, S. Molecular Doping in FewMolecule Polymer-Dopant Complexes Shows Reduced Coulomb Binding. J. Mater. Chem. C 2020, 8, 1192911935.

23 Dong, C.-D.; Schumacher, S.Microscopic Insights into Charge Formation and Energetics in n-Doped Organic Semiconductors. J. Phys. Chem. C 2021, 125, 2182421830.

24 Fazzi, D.; Fabiano, S.; Ruoko, T.-P.; Meerholz, K.; Negri, F. Polarons in $\pi$-conjugated ladder-type polymers: a broken symmetry density functional description. J. Mater. Chem. C 2019, 7, 12876-12885.

25 Zamadar, M.; Asaoka, S.; Grills, D. C.; Miller, J. R. Giant Infrared Absorption Bands of Electrons and Holes in Conjugated Molecules. Nat. Commun. 2013, 4, 2818.

${ }^{26}$ Frisch, M. J.; Trucks, G. W.; Schlegel, H. B.; Scuseria, G. E.; Robb, M. A.; Cheeseman, J. R.; Scalmani, G.; Barone, V.; Mennucci, B.; Petersson, G. A.; Nakatsuji, H.; et al. Gaussian 09, revision D.01; Gaussian, Inc.: Wallingford, CT, 2009.

27 Lu, T.; Chen, F. Multiwfn: a Multifunctional Wavefunction Analyzer., J. Comput. Chem. 2012, 33, 580-592.

28 Zaikowski, L.; Kaur, P.; Gelfond, C.; Selvaggio, E.; Asaoka, S.; Wu, Q.; Chen, H.-C.; Takeda, N.; Cook, A. 
R.; Yang, A.Polarons, Bipolarons, and Side-by-Side Polarons in Reduction of Oligofluorenes. J. Am. Chem. Soc. 2012, 134, 10852-10863.

29 Anderson, M.; Ramanan, C.; Fontanesi, C.; Frick, A.; Surana, S.; Cheyns, D.; Furno, M.; Keller, T.; Allard, S.; Scherf, U. et al. Displacement of polarons by vibrational modes in doped conjugated polymers. Phys. Rev. Mater. 2017, 1, 55604 .

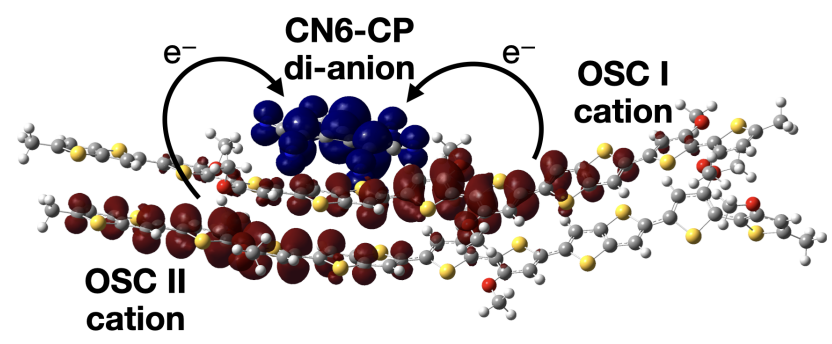

FIG. 7. TOC figure. 